\title{
NtMYB4 and NtCHS1 Are Critical Factors in the Regulation of Flavonoid Biosynthesis and Are Involved in Salinity Responsiveness
}

\section{OPEN ACCESS}

Edited by:

Francesco Paolocci,

Institute of Bioscience

and Bioresources, National Research

Council, Italy

Reviewed by:

Pedro Carrasco,

University of Valencia, Spain

Andrés Gárriz,

Consejo Nacional de Investigaciones Cientificas y Técnicas (CONICET),

Argentina

*Correspondence:

Shuai Chen

chenshuai019@126.com

Aiguo Yang

yangaiguo@caas.cn

Specialty section:

This article was submitted to

Plant Metabolism

and Chemodiversity,

a section of the journal

Frontiers in Plant Science

Received: 22 November 2018 Accepted: 05 February 2019

Published: 21 February 2019

Citation:

Chen S, Wu F, Li Y, Qian Y, Pan X, Li F, Wang Y, Wu Z, Fu C, Lin H and Yang A (2019) NtMYB4 and NtCHS1 Are Critical Factors in the Regulation

of Flavonoid Biosynthesis and Are Involved in Salinity Responsiveness.

Front. Plant Sci. 10:178.

doi: 10.3389/fpls.2019.00178

\section{Shuai Chen ${ }^{1 *}$, Fengyan Wu ${ }^{1}$, Yiting Li ${ }^{1}$, Yanli Qian ${ }^{1}$, Xuhao Pan ${ }^{1}$, Fengxia Li', Yuanying Wang ${ }^{1}$, Zhenying $W_{u^{2}}$, Chunxiang $\mathrm{Fu}^{2}$, Hao Lin ${ }^{3}$ and Aiguo Yang ${ }^{1 *}$}

\footnotetext{
${ }^{1}$ Tobacco Research Institute, Chinese Academy of Agricultural Sciences, Qingdao, China, ${ }^{2}$ Qingdao Institute of Bioenergy and Bioprocess Technology, Chinese Academy of Sciences, Qingdao, China, ${ }^{3}$ Biotechnology Research Institute, Chinese Academy of Agricultural Sciences, Beijing, China
}

High levels of salinity induce serious oxidative damage in plants. Flavonoids, as antioxidants, have important roles in reactive oxygen species (ROS) scavenging. In the present study, the tobacco R2R3 MYB type repressor, NtMYB4, was isolated and characterized. The expression of NtMYB4 was suppressed by salinity. Overexpression of NtMYB4 reduced the salt tolerance in transgenic tobacco plants. NtMYB4 repressed the promoter activity of $\mathrm{NtCHS1}$ and negatively regulated its expression. Rutin accumulation was significantly decreased in NtMYB4 overexpressing transgenic plants and NtCHS1 RNAi silenced transgenic plants. Moreover, high $\mathrm{H}_{2} \mathrm{O}_{2}$ and $\mathrm{O}_{2}^{-}$contents were detected in both types of rutin-reduced transgenic plants under high salt stress. In addition, exogenous rutin supplementation effectively scavenged $\mathrm{ROS}\left(\mathrm{H}_{2} \mathrm{O}_{2}\right.$ and $\left.\mathrm{O}_{2}^{-}\right)$and improved the salt tolerance of the rutin-reduced transgenic plants. In contrast, $\mathrm{NtCHS1}$ overexpressing plants had increased rutin accumulation, lower $\mathrm{H}_{2} \mathrm{O}_{2}$ and $\mathrm{O}_{2}^{-}$contents, and higher tolerance to salinity. These results suggested that tobacco NtMYB4 acts as a salinity response repressor and negatively regulates NtCHS1 expression, which results in the reduced flavonoid accumulation and weakened ROS-scavenging ability under salt stress.

Keywords: NtMYB4, NtCHS1, flavonoid pathway, ROS level, salt stress

\section{INTRODUCTION}

Salinity is one of the most significant abiotic stresses affecting plant growth and agricultural productivity. The response of plants to salinity is a complex set of traits that involves signaling and metabolic processes at molecular, cellular, and whole-plant levels. Extensive research has been carried out on the mechanisms of plant salt tolerance, including ion homeostasis, osmotic adjustment, reactive oxygen species (ROS) scavenging, detoxification, signal transduction, transcription, and crosstalk with other stresses (Tuteja, 2007; Zhang et al., 2012; Deinlein et al., 2014). However, the detailed mechanism underlying plant salt tolerance is unclear.

The exposure of plants to salinity results in massive changes in gene expression. Transcription factors (TFs) are initially vital in sensing salt and stimulating tolerance responses 
(Deinlein et al., 2014). TFs, including MYB, WRKY, bHLH, bZIP, and NAC families, are differentially expressed in response to salinity (Jiang and Deyholos, 2009; Jiang et al., 2009; Yang et al., 2009; He et al., 2012; Cui et al., 2013; Deinlein et al., 2014). These TFs regulate the expression levels of various genes that may function in salt tolerance of plants. Studies have demonstrated that gene manipulation could alter the salt tolerance of plants. For example, the ectopic expression of TaMYB73 from wheat in Arabidopsis thaliana activates the expression of stress signaling genes such as AtCBF3 and AtABF3, and enhances the tolerance to ionic and salinity stress (He et al., 2012). In turn, the TF AtbZIP24, which down-regulates the expression of stress inducible-genes such as the $\mathrm{Na}^{+}$transporter AtHKT1; 1, is required during salt stress in plants. In this trend, AtbZIP24 silenced transgenic Arabidopsis shows increased salt tolerance compared to wild type (WT) plants (Yang et al., 2009). In the same line, the ethylene responsive factor BpERF11 down-regulates the expression of important genes involved in abiotic stress tolerance such as LEA and DHN, and leads to reduced levels of proline and the accumulation of ROS. Thus, BpERF11 negatively regulates plant salt and osmotic tolerance (Zhang et al., 2016).

Flavonoids are important secondary metabolites in plants and have important roles in the resistance to oxidative damage caused by ROS during plant growth and abiotic stresses due to their antioxidant activity (Moore et al., 2005; Yu and Jez, 2008; Agati et al., 2012; Pandey et al., 2015). It has been reported that flavonols modulate ROS levels to control the stomata aperture (Watkins et al., 2017) and the formation of root hairs (Maloney et al., 2014). Accumulation of flavonoids, such as flavonols, anthocyanins, and proanthocyanidins in plants could enhance salt tolerance (Liu et al., 2014; Li P. et al., 2017). In this trend, moderate salt stress was shown to induce the accumulation of flavonoids and promote the quality of agricultural crops and medicinal plants (Lim et al., 2012; Colla et al., 2013). Rutin is a glycoside flavonoid that is abundant in a wide variety of plants. Rutin has received much attention due to its health benefits, such as activity against diabetes and inflammation, prevention of hypertension, and antioxidant properties (Kerdudo et al., 2014; Yoo et al., 2014; Kaur and Muthuraman, 2016; Ghorbani, 2017). Rutin biosynthesis is regulated by flavonoid structural genes and TFs. Six structural genes, $P A L, C 4 H, 4 C L, C H S, C H I$, and FLS, are the major genes involved in the rutin biosynthetic pathway (WinkelShirley, 2001). Rutin is one of the most abundant flavonoids, accounting for more than $90 \%$ of total flavonoids in tobacco ( $\mathrm{Li}$ et al., 2014). For this reason, tobacco is the proper model plant to explore the function of rutin in salt tolerance.

Increasing evidence has indicated that R2R3 MYB TFs play important roles in regulating the flavonoid biosynthetic pathway in plants (Dubos et al., 2010; Zhang et al., 2014; Zhou et al., 2017a). R2R3 MYB subgroup 4 TFs, AtMYB3, AtMYB4, AtMYB7, and AtMYB32 are involved in repression of the phenylpropanoid and flavonoid pathways in Arabidopsis (Jin et al., 2000; Dubos et al., 2010; Fornalé et al., 2014; Zhou et al., 2017b). Recently, many genes orthologous to AtMYB4, including ZmMYB31, ZmMYB42, EgMYB1, BrMYB4, CsMYB4a, and $F t M Y B 11$, were identified from different plants, all of which negatively regulate phenylpropanoid biosynthesis (Fornalé et al., 2006; Legay et al., 2010; Zhang et al., 2014; Li M. et al., 2017; Zhou et al., 2017a). To the best of our knowledge, tobacco MYB repressors have not been studied yet.

In the present study, we identified an R2R3 MYB TF, NtMYB4, which is orthologous to AtMYB4. We determined that the expression of the NtMYB4 gene was significantly repressed by high salinity. In addition overexpression of NtMYB4 resulted in reduced rutin accumulation, which affects the antioxidant ability of plants under salt stress. Our results indicate that the reduced rutin contents might be a consequence of the repression of the biosynthetic gene NtCHS1 by NtMYB4. As a whole, these data indicate that NtMYB4 plays an important function during the responses of plant to saltine stress.

\section{MATERIALS AND METHODS}

\section{NtMYB4 Gene Isolation}

A search for sequences orthologous to AtMYB4 was conducted using the Blastp tool in China Tobacco Genome Database ${ }^{1}$. The isolated MYB protein sequences were aligned and MYB proteins containing the conserved LLsrGIDPxT/SHRxI/L, EAR repression, zinc-finger $\left(\mathrm{CX}_{1-2} \mathrm{CX}_{7-12} \mathrm{CX}_{2} \mathrm{C}\right)$, and GY/FDFLGL motifs in the C-termini were selected for further analysis (Jin et al., 2000; Zhou et al., 2015). PCR was used to clone the full length cDNA of NtMYB4 with the primers NtMYB4-F/NtMYB4R (Supplementary Table S1). The PCR products were purified and cloned with a pEASY-Blunt Cloning Kit (TransGen Biotech, China) and then sequenced. RNA isolation from tobacco leaves, cDNA synthesis, and PCR were performed as described in Chen et al. (2017). Protein sequences were aligned in DNAMAN software (Lynnon Biosoft, United States). A phylogenetic tree was constructed with the neighbor-joining (NJ) algorithm in the MEGA6.0 program (Kumar et al., 2008). The robustness of the tree topology was assessed using 1,000 bootstrap replicates.

\section{Genetic Construction and Plant Transformation}

For the preparation of cassettes for the overexpression of $N t M Y B 4$ and NtCHS1 and for the silencing of NtCHS1, each gene-specific amplicon was amplified from tobacco leaf cDNA, synthetized as reported in the following paragraph. In order to construct the overexpression vector NtMYB4-pCHF1, the entire coding sequence (CDS) of NtMYB4 was amplified using primers NtMYB4OE-F/NtMYB4OE-R (Supplementary Table S1). The PCR product was digested with $\mathrm{XbaI} / \mathrm{SacI}$ and cloned into the XbaI/SacI digested pCHF1 plasmid under the control of the CaMV 35S promoter. To construct the overexpression vector NtCHS1-pC3301-ZDS, coding region (CDS) of NtCHS1 (Chen et al., 2017) was amplified using primers NtCHS1OE-F/NtCHS1OE-R (Supplementary Table S1). The $\mathrm{PCR}$ product was digested with $\mathrm{XbaI} / \mathrm{SacI}$ and cloned into the XbaI/SacI digested pC3301-ZDS plasmid under the control of the CaMV 35S promoter. A 293 bp long fragment

\footnotetext{
${ }^{1}$ www.tobaccodb.org
} 
of NtCHS1 was amplified using the gene-specific primers NtCHS1RNAi-F/NtCHS1RNAi-R, which contain attB sites (underlined) (Supplementary Table S1). Using BP and LR reactions, the target fragment was then ligated into the destination vector pH7GWIWG2(I) (Xu et al., 2011) according to the Gateway ${ }^{\mathrm{TM}}$ manufacturer's instructions (Invitrogen, Carlsbad, CA, United States), to yield the NtCHS1-RNA $i$ vector. The pCHF1-NtMYB4, pC3301-ZDS-NtCHS1 and NtCHS1-RNAi vectors were introduced into Agrobacterium tumefaciens strain LBA4404. Transformation of tobacco was performed using the leaf disc method as reported by Horsch et al. (1985).

In order to construct the subcellular localization vector NtMYB4-CPB-YFP, NtMYB4-YFP-F/NtMYB4-YFP-R primers (Supplementary Table S1) were used to amplify the NtMYB4 coding region and the PCR product was cloned into the BamHI digested pCPB-YFP plasmid using the ClonExpress ${ }^{\circledR}$ Entry One Step Cloning Kit (Vazyme Biotech, China).

With the purpose of constructing the reporters and effectors for UAS/GAL4-based transcriptional repression assay. NtMYB4BD-F/NtMYB4BD-R primers (Supplementary Table S1) were used to amplify the NtMYB4 coding region. For constructing the pGBKT7-NtMYB4 vector, the PCR product was cloned into the BamHI digested pGBKT7 plasmid using the ClonExpress $^{\circledR}$ Entry One Step Cloning Kit (Vazyme Biotech, China). To construct the effector vectors, two primer pairs GAL4BD-F/GAL4BD-R and GAL4BD-F/PMDC32-NtMYB4-R (Supplementary Table S1) were used to amplify the fusion sequence from pGBKT7-NtMYB4. Then the fusion sequences were cloned into the BamHI digested pMDC32 plasmid using the ClonExpress ${ }^{\circledR}$ Entry One Step Cloning Kit. The reporters 35S-UAS::GUS were constructed according to the method of Chen et al. (2014).

With the purpose of constructing the reporters and effectors for the dual luciferase assay, seven primer pairs pPAL-F/pPAL$\mathrm{R}, \mathrm{pC} 4 \mathrm{H}-\mathrm{F} / \mathrm{pC} 4 \mathrm{H}-\mathrm{R}, \mathrm{p} 4 \mathrm{CL}-\mathrm{F} / \mathrm{p} 4 \mathrm{CL}-\mathrm{R}, \mathrm{pCHS1-F} / \mathrm{pCHS} 1-\mathrm{R}$, pCHI-F/pCHI-R, pFLS-F/pFLS-R, and pANS-F/pANS-R (Supplementary Table S1) were designed to amplify the promoter sequences of the genes NtPAL, NtC4H, Nt4CL, NtCHS1, NtCHI, NtFLS, and NtANS, respectively. For pGreen vectors construction, the promoter sequences of seven genes were cloned into the BamHI digested pGreen plasmid using the ClonExpress $^{\circledR}$ Entry One Step Cloning Kit.

\section{Quantitative RT-PCR Analysis (qRT-PCR)}

Total RNA was extracted from WT tobacco tissue, such as leaves, roots, stems, buds and flowers, and from transgenic tobacco leaves according to the method described in Chen et al. (2017). First-strand cDNA was synthesized using a PrimeScript ${ }^{\mathrm{TM}}$ RT Master Mix (Perfect Real Time) (Takara, Clontech, Japan) according to the manufacturer's instructions. The qRT-PCR was performed using SYBR ${ }^{\circledR}$ Premix Ex Taq ${ }^{\mathrm{TM}}$ (Tli RNaseH Plus) (Takara, Clontech, Japan), and primers listed in Supplementary Table S1. The Tob103 gene (GenBank accession no. U60495) served as an internal control. The reaction mixtures consisted in $10 \mu \mathrm{l}$ SYBR Green mix, $0.4 \mu \mathrm{l}$ forward qRT-PCR primer, $0.4 \mu \mathrm{l}$ reverse primer, $1 \mu \mathrm{l}$ template $\mathrm{cDNA}$, and $7.8 \mu \mathrm{l}$ sterile water. The thermal cycling parameters were $95^{\circ} \mathrm{C}$ for $30 \mathrm{~s}$, followed by 40 cycles of $95^{\circ} \mathrm{C}$ for $15 \mathrm{~s}$ and $58^{\circ} \mathrm{C}$ for $34 \mathrm{~s}$. The relative expression levels were normalized to the expression of the Tob103 gene. The comparative cycle threshold $(\Delta \Delta \mathrm{CT})$ method was used to calculate the relative expression levels of the target genes. Data were expressed as the mean $\pm \mathrm{SD}$ as determined from three independent biological replicates.

\section{Subcellular Localization}

NtMYB4-CPB-YFP construct was transformed into A. tumefaciens EHA105 strain. A single bacterial clone was grown at $28^{\circ} \mathrm{C}$ overnight, centrifuged for $10 \mathrm{~min}$ at 4,200 rpm, re-suspended in $10 \mathrm{mM} \mathrm{MgCl}_{2} / 10 \mathrm{mM}$ MES to a final OD = 1.0 and cultured for $2-3 \mathrm{~h}$ with $2 \mu \mathrm{l} 100 \mathrm{mM}$ acetosyringone in the dark. Transformed bacteria were infiltrated into leaves of Nicotiana benthamiana (Chen et al., 2014). The leaves were collected between 48 and $72 \mathrm{~h}$ after infiltration and examined by confocal laser scanning microscopy. Images of triplicate infiltrated leaves were acquired with a Fluo View ${ }^{\mathrm{TM}}$ FV1000 microscope equipped with an argon laser line of $515 \mathrm{~nm}$ (excitation) for YFP signal.

\section{Dual Luciferase Assay}

The binding to promoters of flavonoid structural genes was assessed to test the transcriptional regulation activity of NtMYB4. Five promoter-pGreen vectors, pCHF1-NtMYB4, pCHF1 empty vector, and P19 were transformed into A. tumefaciens AH105. The bacteria were grown with $2 \mu 1100 \mathrm{mM}$ acetosyringone at $28^{\circ} \mathrm{C}$ overnight. To prepare the suspension used for infiltration, bacteria were re-suspended in $10 \mathrm{mM} \mathrm{MgCl} / 10 \mathrm{mM}$ MES to a final $\mathrm{OD}=1.0$. Transformed bacteria containing pGreen, pCHF1 and P19 vectors were combined at a 3:3:1 volume ratio, cultured for 1-2 h in the dark and then infiltrated into leaves of $N$. benthamiana as reported above. The leaves were harvested for dual luciferase assay analysis at $72 \mathrm{~h}$ after infiltration according to the instructions of the Dual-Luciferase ${ }^{\circledR}$ Reporter Assay System (Promega, United States).

\section{UAS/GAL4-Based Transcriptional Repression Assay}

A transient assay was carried out to test NtMYB4 transcriptional repression activity. To prepare the suspension used for infiltration, bacteria were re-suspended in $10 \mathrm{mM} \mathrm{MgCl}_{2} / 10 \mathrm{mM}$ MES to a final OD $=1.0$. Transformed bacteria containing 35SUAS::GUS, GAL4BD/GAL4BD-NtMYB4 and P19 vectors were combined at a 2:2:1 volume ratio and cultured for $2-3 \mathrm{~h}$ in the dark. Transformed bacteria were infiltrated into leaves of $N$. benthamiana and cultured under weak light conditions. The leaves were harvested for GUS staining at $72 \mathrm{~h}$ after infiltration (Liu et al., 2010).

\section{Plant Growth and Salt Treatment}

Seeds of WT and transgenic plants of Nicotiana tabacum cultivar Honghuadajinyuan (HD) were sterilized in 75\% ethanol for $30 \mathrm{~s}$ and then washed three times in sterile water. After that, they were further sterilized in $15 \%(\mathrm{w} / \mathrm{v}) \mathrm{H}_{2} \mathrm{O}_{2}$ solution for $8 \mathrm{~min}$ and then washed three times in sterile water. For the germination 
test, the sterilized seeds were cultured on MS solid medium with $0 \mathrm{mM} \mathrm{NaCl}, 200 \mathrm{mM} \mathrm{NaCl}$, or $200 \mathrm{mM} \mathrm{NaCl}+100 \mu \mathrm{M}$ rutin, respectively. The germination rate was measured in the next $0-$ 11 days after sowing. For the root length test, the sterilized seeds were cultured on 1/2 MS solid medium. Ten day-old seedlings were then transferred to MS solid medium with $0 \mathrm{mM} \mathrm{NaCl}$, $200 \mathrm{mM} \mathrm{NaCl}$, or $200 \mathrm{mM} \mathrm{NaCl}+100 \mu \mathrm{M}$ rutin. The plates were cultured in growth chambers under a $16 \mathrm{~h}$ light $/ 8 \mathrm{~h}$ dark photoperiod at $28^{\circ} \mathrm{C}$. Root length was measured at 20 days after transfer. Forty seeds were used per experiment, and all experiments were repeated three times independently. For the analysis of NtMYB4 expression under salt stress, seedlings with three or four leaves grown on basal MS medium were transferred to a liquid MS medium containing $200 \mathrm{mM} \mathrm{NaCl}$ for 12 or $24 \mathrm{~h}$. The corresponding control was prepared by incubating seedlings in MS liquid medium for the same time. Leaves were harvested after $0,12,24 \mathrm{~h}$, and 30 days of treatments according to the method of Chen et al. (2017). All collected samples were frozen in liquid nitrogen and stored at $-80^{\circ} \mathrm{C}$.

\section{Measurements of $\mathrm{H}_{2} \mathrm{O}_{2}$ and $\mathrm{O}_{2}^{-}$Content}

Collected leaves of 1-month-old plants treated with high salt stress $(200 \mathrm{mM} \mathrm{NaCl})$ were used to measure ROS levels. The measurement of $\mathrm{H}_{2} \mathrm{O}_{2}$ content was based on the method described by Libik et al. (2005). The $\mathrm{O}_{2}^{-}$content was determined by measuring the formation of red azo compound, which has specific absorption peak at $530 \mathrm{~nm}$, according to the $\mathrm{O}_{2}^{-}$Detection Kit (SA-1-G, Keming, China). In addition, $\mathrm{H}_{2} \mathrm{O}_{2}$ and $\mathrm{O}_{2}^{-}$were detected visually using the 3'3'-diaminobenzidine (DAB) and nitro blue tetrazolium (NBT) histochemical staining method as described by Zhao et al. (2016). For measurements of each treatment, leaves were collected from at least three plants.

\section{Measurement of Rutin by High- Performance Liquid Chromatography}

Rutin was extracted according to the method described by $\mathrm{Li}$ et al. (2015), with some modifications. Dried leaves samples $(0.1 \mathrm{~g})$ were extracted with $1 \mathrm{ml}$ of $80 \%$ ethanol by $20 \mathrm{~min}$ at $40^{\circ} \mathrm{C}$ with ultrasound, followed by a new extraction with $95 \%$ ethanol for $10 \mathrm{~min}$, and then three washes with $80 \%$ ethanol $10 \mathrm{~min}$. Supernatants were collected and the volume brought to $50 \mathrm{ml}$ by distilled water and filtered in a $0.45 \mu \mathrm{m}, 13 \mathrm{~mm}$ Millex Syringe Filter (Merck Millipore, Carrigtwohill Co., Cork, Ireland). Rutin contents in extracts were analyzed by HPLC as described previously (Huang et al., 2016). Analysis was carried out on three independent biological replicates each containing three technical replicates.

\section{Measurement of Anthocyanin Content}

The flowers were collected in the full-blossom period of WT and NtCHS1 transgenic tobacco. Anthocyanin from flowers were measured by spectrophotometry according to the method of Pattanaik et al. (2010). All samples were measured as triplicates in three independent biological replicates.

\section{Statistical Analysis}

Data were analyzed by Duncan's multiple range tests in the ANOVA program of SPSS (IBM SPSS 22). The $P$-value less than 0.05 and 0.001 was considered statistically significant.

\section{RESULTS}

\section{Characterization of NtMYB4, a Nuclear Localized Transcription Repressor}

By blasting the tobacco genome database with AtMYB4 amino acid sequence, we identified a R2R3 MYB, NtMYB4, as orthologous to AtMYB4. An alignment of amino acid sequences of NtMYB4 and three other orthologous sequences revealed that it contained the R2 and R3 conserved motifs in the N-terminal region, and the conserved LLsrGIDPxT/SHRxI/L motif, EAR repression motif, zinc-finger domain $\left(\mathrm{CX}_{1-2} \mathrm{CX}_{7-12} \mathrm{CX}_{2} \mathrm{C}\right)$, and GY/FDFLGL motif in the C-terminal region (Supplementary Figure S1). A phylogenetic analysis was performed using the amino acid sequences of NtMYB4 and 10 other orthologous sequences. The result indicated that NtMYB4 clustered with AtMYB4, AtMYB7, and AtMYB32 (Figure 1B), which are members of subgroup 4 and repressors of the phenylpropanoid pathway. Subcellular localization analysis showed that NtMYB4 was a nuclear localized protein (Figure 1A). Spatial transcript accumulation analysis showed that in seedlings NtMYB4 was more expressed in leaves, whereas in mature plants in all organs assayed, especially the floral bud (Figure 1C). A UAS/GAL4based transcriptional repression assay showed that NtMYB4 had strong repression activity in tobacco leaves (Figure 1D). These results suggested that NtMYB4 might act as a nuclear localized transcription repressor in tobacco.

\section{NtMYB4 Overexpression Significantly Reduced Salt Tolerance}

Real-time PCR was performed to determine changes in expression levels of NtMYB4 in WT tobacco in response to high salt stress. The result showed that the level of NtMYB4 mRNA in leaves was significantly lower after 12 and $24 \mathrm{~h}$ of seedling treatment with $200 \mathrm{mM} \mathrm{NaCl}$ (Figure 2A).

To verify the function of NtMYB4 in salt tolerance, we overexpressed the NtMYB4 gene in tobacco. Three transgenic lines M4OE-7, M4OE-9, and M4OE-28 were obtained (Figure 2B) and analyzed in detail. The germination rate and root length of WT and transgenic seeds under high salt stress treatment were measured. Even though there were no obvious differences in germination rates between $\mathrm{WT}$ and transgenic seeds when grown on basal MS medium $(0 \mathrm{mM} \mathrm{NaCl})$, severe suppression was observed in NtMYB4 overexpressing transgenic seeds under $200 \mathrm{mM} \mathrm{NaCl}$ as the germination rate was decreased 92\% (Figure 2C). Moreover, we also observed that salt stress provoked a delay in germination, which normally occurs at the fourth day. Thus, WT seeds started to germinate on the sixth day in MS amended with $200 \mathrm{mM} \mathrm{NaCl}$, while the NtMYB4 overexpressing transgenic seeds on the ninth day (Figure 2D). On basal medium, no obvious differences in 


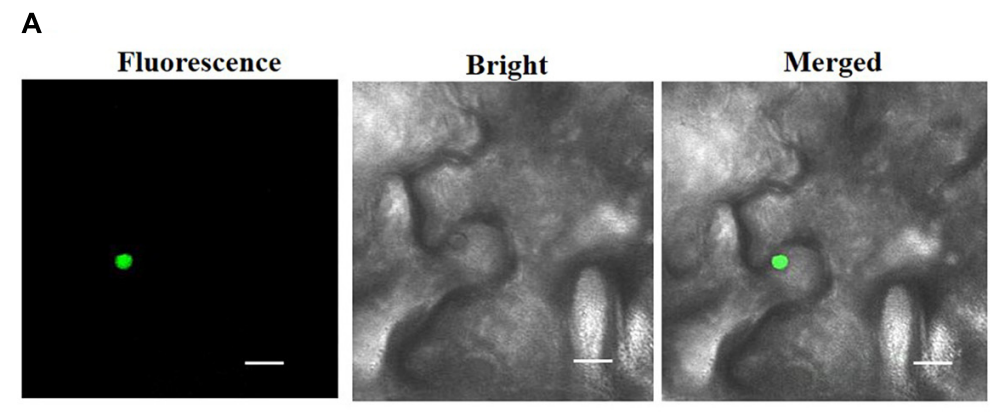

B

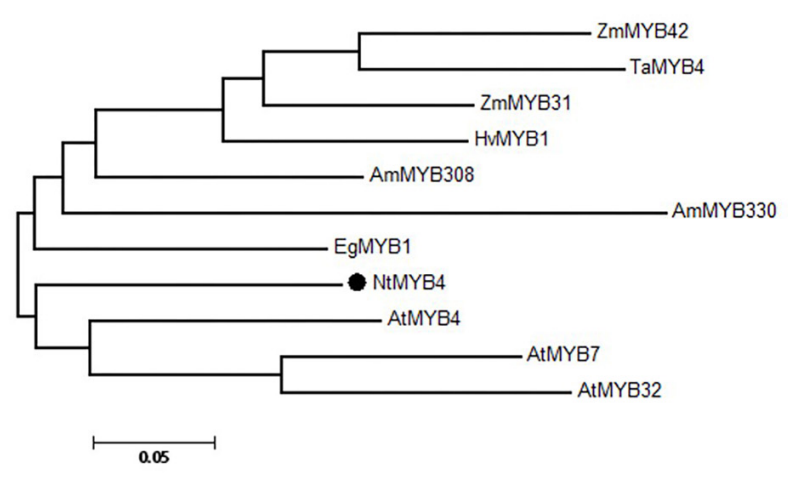

C

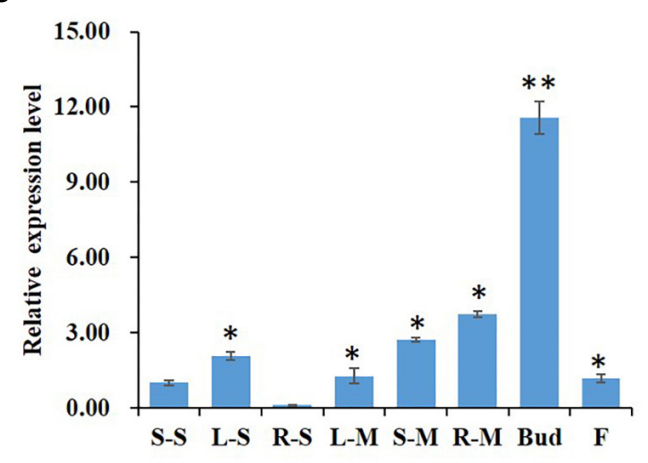

D

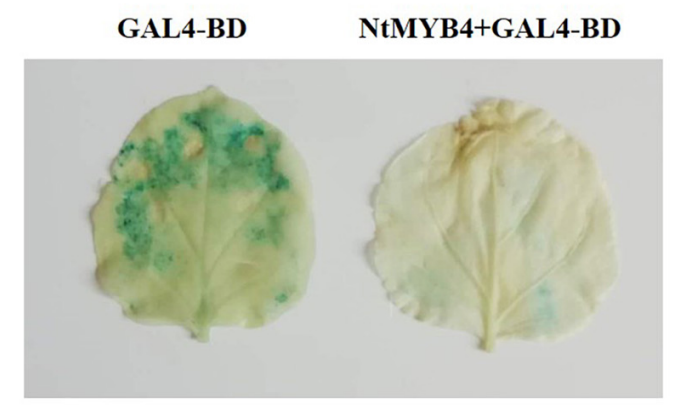

FIGURE 1 | NtMYB4 is a nuclear localized transcription repressor in tobacco. (A) Subcellular localization analysis of NtMYB4. An NtMYB4-GFP construct was transformed into leaves of $\mathrm{N}$. benthamiana and examined by confocal laser scanning microscopy. A confocal micrograph is shown at the left (green fluorescent protein, GFP), the corresponding differential interference contrast (bright) image is in the middle, and the merged image is at the right. Bars, $20 \mu \mathrm{m}$. (B) Phylogenetic tree based on the amino acid sequences of NtMYB4 and 10 other R2R3 MYB subgroup 4 members. The NtMYB4 isolated in this study is highlighted by a black dot. Accession numbers for MYB subgroup 4 sequences are: AmMYB308 (JQ0960), AmMYB330 (P81395), AtMYB4 (AY519615), AtMYB7 (AEC06531), AtMYB32 (NP_195225), EgMYB1 (CAE09058), HvMYB1 (P20026), TaMYB4 (AAT37167), ZmMYB31 (CAJ42202), and ZmMYB42 (CAJ42204). (C) The expression pattern of NtMYB4 in tobacco root, stem, leaf flower and bud was detected by qRT-PCR. Expression data were detected in two representative growth stages of common tobacco, including the seedling $(S)$ and mature $(M)$ stages. Organs sampled included leaf $(L)$, stem $(S)$, root $(R)$, flower $(F)$. Asterisks in (C) indicate differences between tissues $\left({ }^{*} P<0.05,{ }^{* *} P<0.01\right)$. (D) UAS/GAL4-based transcriptional repression assay of NtMYB4 in $N$. benthamiana leaves. NtMYB4 repressed the 35S-driven GUS activity. Pairwise combinations of constructs as indicated were co-infiltrated into tobacco leaves and stained for GUS activity. The left leaf marked GAL4-BD is 35S-driven GAL4 DNA binding domain (BD) as control; the right leaf marked NtMYB4+GAL4-BD was 35S-driven full-length NtMYB4 fused to the Gal4-BD domain.

seedling growth and root length were observed between WT and transgenic plants (Figure 2F). Conversely, $200 \mathrm{mM} \mathrm{NaCl}$ treatment caused a significant inhibition in root elongation in both WT and transgenic lines, being 53, 87, 86, and $86 \%$ the inhibition observed in WT, M4OE-7, M4OE-9, and M4OE28 , respectively. Interestingly, under salt treatment, the root length was significantly reduced in all transgenic lines with respect to WT plants (Figures 2E,F). These results showed that overexpression of NtMYB4 reduced the seed germination rate and root elongation under $200 \mathrm{mM} \mathrm{NaCl}$ compared to WT, which indicated that NtMYB4 plays a negative role in plant salt tolerance.

\section{NtMYB4 Overexpression Leads to Reduced Flavonoid Accumulation}

Numerous reports have demonstrated that salt stress can induce the accumulation of phenolic compounds in plant tissues (Ksouri et al., 2007; Baâtour et al., 2013). The key structural genes of the phenylpropanoid pathway $P A L, C H S$, $C H I, D F R, F L S$, and ANS were induced by salt stress in leaves of WT tobacco (Supplementary Figure S2A). Moreover, rutin accumulation increased significantly under $200 \mathrm{mM} \mathrm{NaCl}$ in tobacco (Supplementary Figure S2B). In order to further determine the effects of salt stress in flavonoid biosynthesis, we investigated rutin accumulation in NtMYB4 overexpressing transgenic plants. Rutin content significantly decreased in NtMYB4 overexpressing transgenic tobacco compared to WT (Figure 3A). We speculated that rutin might play a significant role in plant salt tolerance. In order to verify this hypothesis, we supplemented culture media with $100 \mu \mathrm{mol}$ exogenous rutin. Under these experimental conditions, the inhibition of root length elongation was partially counterbalanced (Figures 3B,C), and plants were more robust overall (Figure 3B). Rutin supplementation in fact significantly reduced the inhibition of root length elongation observed both in WT and transgenic 
A

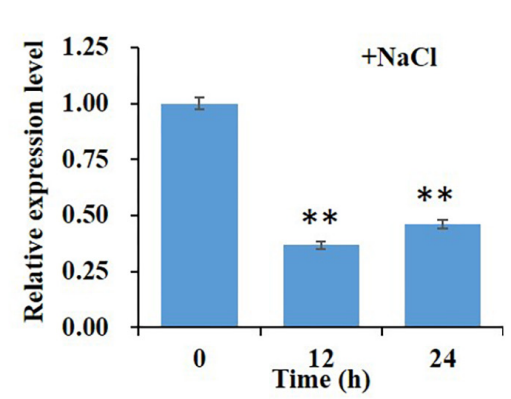

C

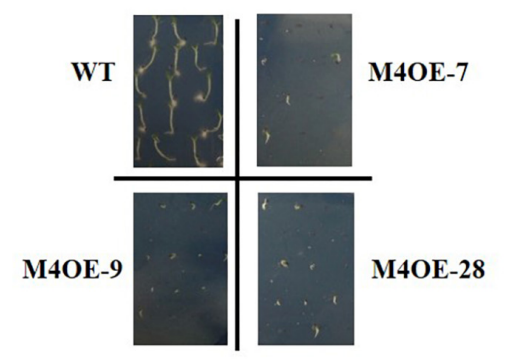

$\mathbf{E}$

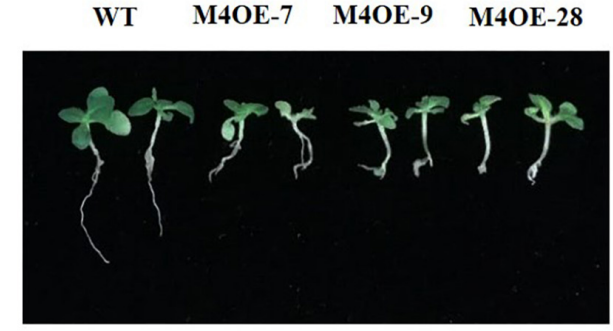

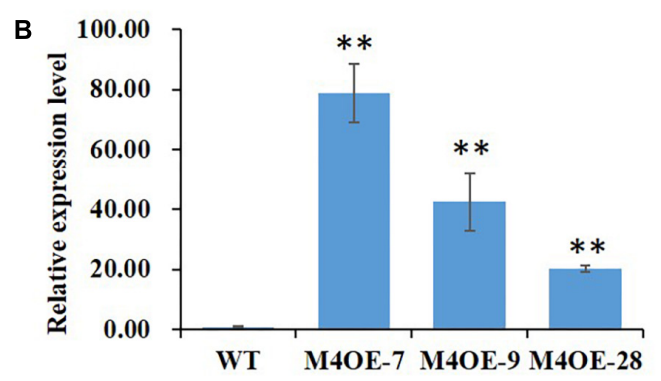

D

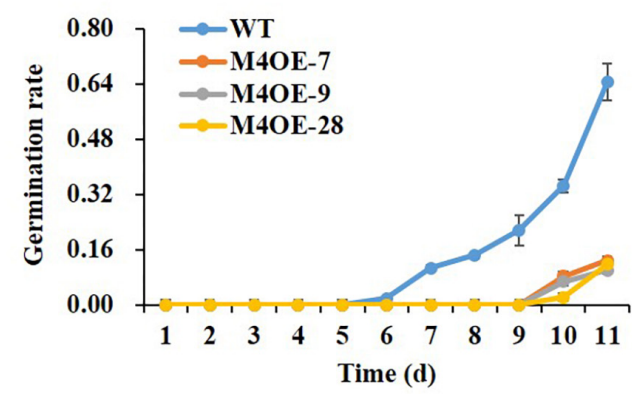

$\mathbf{F}$

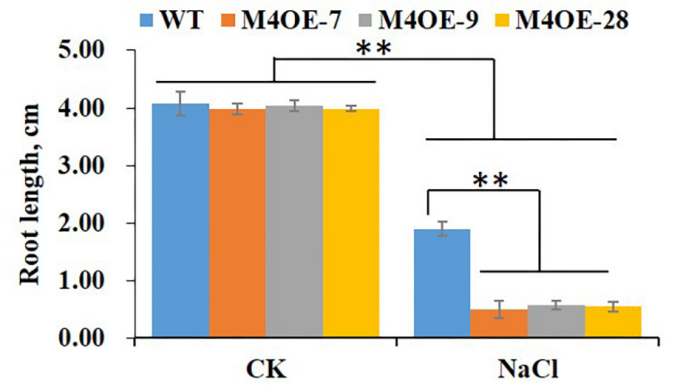

FIGURE 2 | Overexpression of NtMYB4 significantly reduces salt tolerance. The relative expression levels were normalized to the expression of the Tob103 gene (internal control). The relative expression of NtMYB4 in leaves of WT plants was set to 1. (A) The expression of NtMYB4 in leaves of WT plants was significantly suppressed by the addition of $200 \mathrm{mM} \mathrm{NaCl}$ in the medium. Asterisks in (A) indicate significant difference $(* * P<0.01)$ between control condition and salt treatment. (B) The expression level of NtMYB4 in leaves was significantly increased in overexpressing transgenic tobacco compared to the WT. (C,D) The seed germination of WT and three NtMYB4 overexpressing transgenic tobacco lines under $200 \mathrm{mM} \mathrm{NaCl}$ (E,F) The root elongation of WT and three NtMYB4 overexpressing transgenic tobacco lines under $200 \mathrm{mM} \mathrm{NaCl}$. Data are expressed as the mean $\pm \mathrm{SD}$ as determined from three independent biological replicates. Values in (F) that are significantly different between treatments and among lines within each treatment are marked with ${ }^{* *} P<0.01$ and $* P<0.05$.

lines on MS with $200 \mathrm{mM} \mathrm{NaCl}$. Only one, M4OE-9, of the three transgenic lines showed root length similar to WT plants on MS supplemented with $200 \mathrm{mM} \mathrm{NaCl}$ and $100 \mu$ mol rutin (Figure 3C). These result showed that supplementation with rutin enhances the salt tolerance of both WT and NtMYB4 overexpressing transgenic tobacco seedlings.

\section{Rutin Effectively Scavenges ROS Under Salt Stress}

It is well documented that salt stress usually results in the excessive accumulation of ROS, which have deleterious effects on plant cells (Ashraf and Harris, 2004; Torun, 2018). Thanks to their antioxidant capacity, flavonoids play an important role against oxidative injury caused by salt stress (Dixon and Paiva, 1995). To determine the ROS scavenging capability of rutin, seedlings of WT plants with or without $200 \mathrm{mM} \mathrm{NaCl}$ treatment were stained with $\mathrm{DAB}$ or NBT. The histochemical staining suggested that salt stress dramatically increased the $\mathrm{H}_{2} \mathrm{O}_{2}$ or $\mathrm{O}_{2}^{-}$content compared to control. Conversely, supplementation with $100 \mu \mathrm{mol}$ exogenous rutin during salt stress appeared to markedly decrease the levels of ROS (Figure 4A). The content of $\mathrm{H}_{2} \mathrm{O}_{2}$ or $\mathrm{O}_{2}^{-}$was measured using visible spectrophotometry. NtMYB4 overexpressing transgenic plants showed a higher level of $\operatorname{ROS}\left(\mathrm{H}_{2} \mathrm{O}_{2}\right.$ or $\mathrm{O}_{2}^{-}$content $)$than WT plants under salt stress, while exogenous rutin effectively scavenged the ROS (Figures 4B,C). These results showed that rutin plays important roles in scavenging ROS.

\section{NtMYB4 Negatively Regulates the NtCHS1 Activity}

In order to reveal the molecular mechanism mediated by NtMYB4 in regulating flavonoid biosynthesis, we determined the expression levels of flavonoid pathway genes and flavonoid accumulation in WT and NtMYB4 overexpressing transgenic 

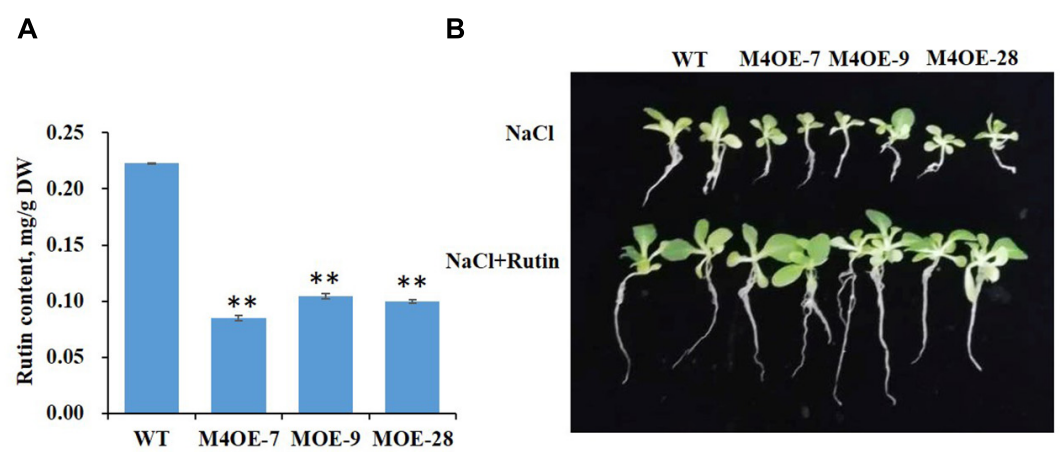

C

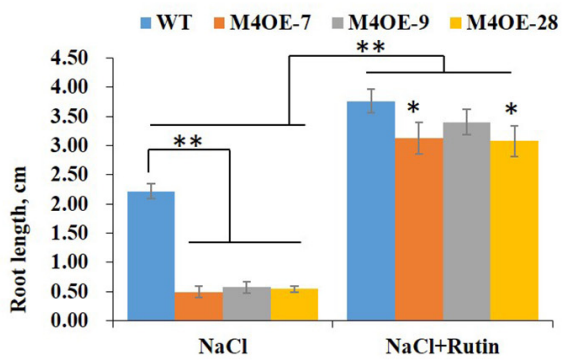

FIGURE 3 | Supplementation with rutin increases salt tolerance of tobacco. (A) Rutin accumulation was significantly decreased in the leaves of NtMYB4 overexpressing transgenic tobacco lines compared to WT. DW, dry weight. Asterisks in (A) indicate significant difference $(* * P<0.01)$ between NtMYB4-OE and WT. (B,C) The root length of WT and three NtMYB4 overexpressing transgenic lines under $200 \mathrm{mM} \mathrm{NaCl}$ with or without $100 \mu \mathrm{M}$ rutin. Data are expressed as the mean \pm SD as determined from three independent biological replicates. Values in (C) that are significantly different between treatments and among lines within each treatment are marked with ${ }^{* *} P<0.01$ and ${ }^{*} P<0.05$.

A

CK

$\mathrm{NaCl}$

$\mathrm{NaCl}+$ Rutin

DAB

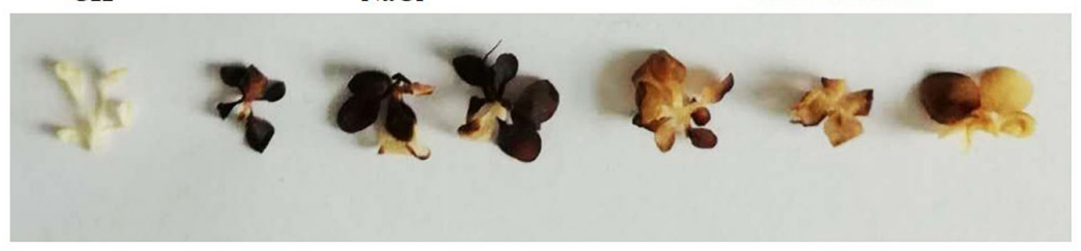

NBT

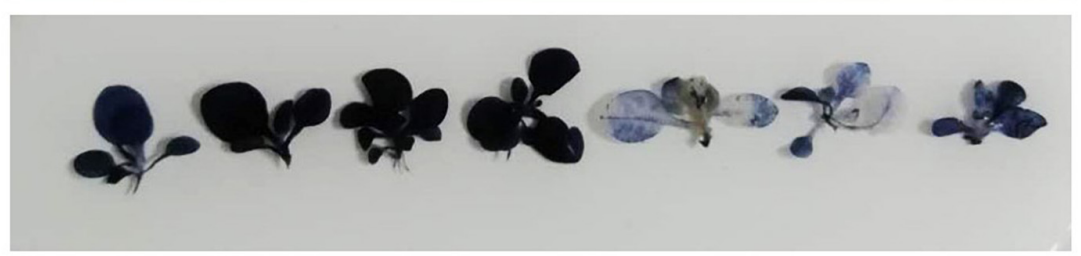

B

$\varpi$ WT $\backsim$ M4OE-7 $\backsim$ M4OE-9 $\backsim$ M4OE-28

C

$\backsim \mathrm{WT} \backsim \mathrm{M} 4 \mathrm{OE}-7 \quad \mathrm{M} 4 \mathrm{OE}-9 \square \mathrm{M} 4 \mathrm{OE}-28$
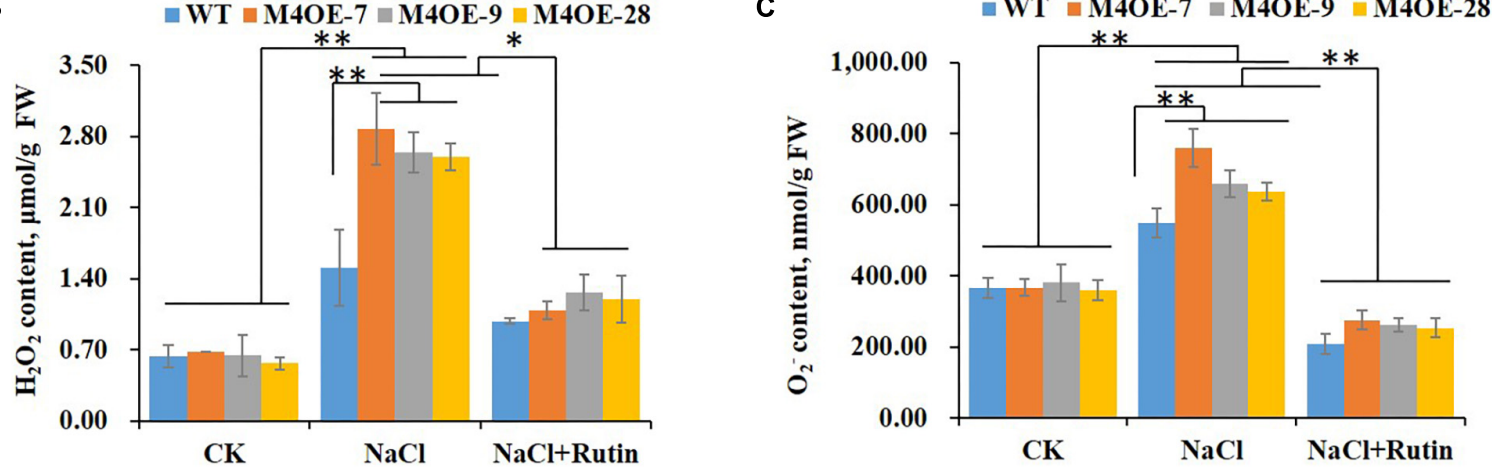

FIGURE 4 | Rutin effectively scavenges the ROS levels under salt stress. (A) DAB or NBT staining of WT plants under control (CK) and salt (NaCl) treatment with or without rutin supplementation. (B,C) $\mathrm{H}_{2} \mathrm{O}_{2}$ or $\mathrm{O}_{2}^{-}$content of WT plants and NtMYB4 overexpressing transgenic lines under control (CK) and salt (NaCl) treatment with or without rutin supplementation, measured by visible spectrophotometry. FW, fresh weight. Data are expressed as the mean \pm SD as determined from three independent biological replicates. Values in $\mathbf{( B , C )}$ that are significantly different between treatments and among lines within each treatment are marked with $* * P<0.01$ and $* P<0.05$.

lines. qRT-PCR result showed that transcript levels of $P A L$, C4H, 4CL, CHS1, FLS, DFR, and ANS were reduced in NtMYB4 overexpressing transgenic plants. Notably, CHS1 and ANS expression was significantly reduced by 94 and $89 \%$, respectively (Figure 5A). Dual-luciferase assay was used to demonstrate whether or not NtMYB4 represses the promoter activity of PAL, 

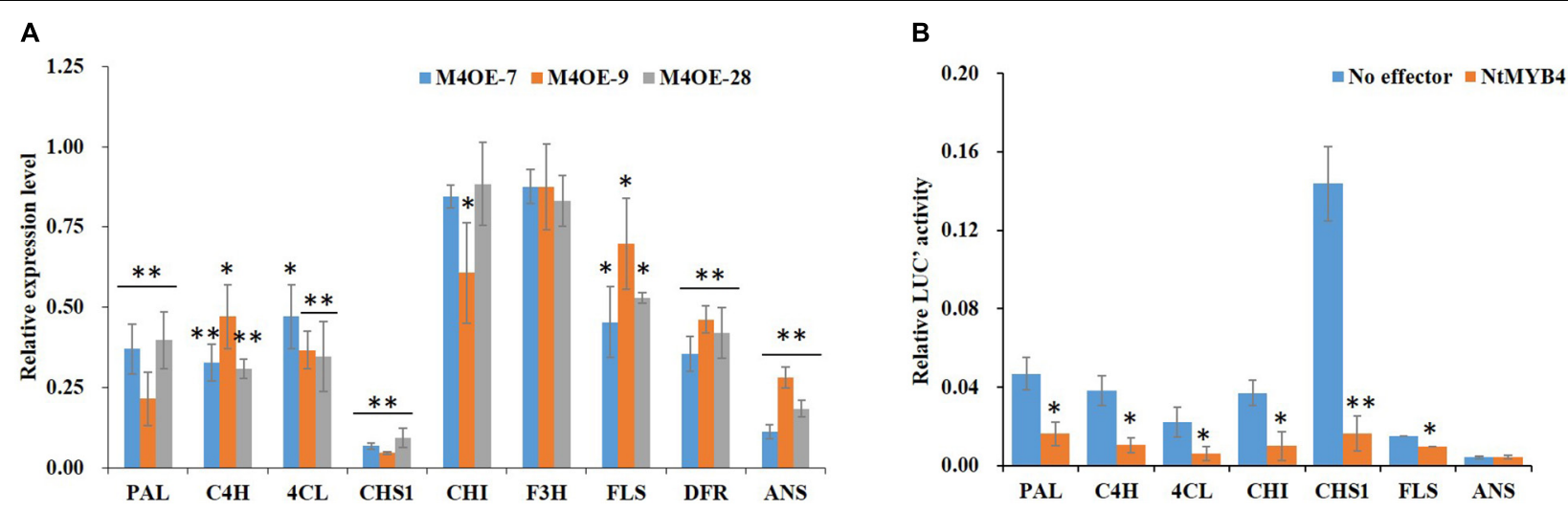

FIGURE 5 | NtMYB4 negatively regulates the expression of flavonoid pathway genes. (A) The expression level of structural genes in NtMYB4 overexpressing transgenic plants were significant inhibited compared to WT. The relative expression of structural genes for the WT control were set to 1. (B) Dual luciferase (LUC) assays of NtMYB4 (effector) and the promoters fused to pGreen luciferase plasmid (reporter). Empty vector was used as the effector in the control assay. The promoters of PAL, C4H, 4CL, CHI, CHS1, FLS, and ANS genes were used in dual luciferase assays $(n=3)$. Data are expressed as the mean \pm SD as determined from three independent biological replicates. Asterisks indicate that the value is significantly different from that of the control $\left({ }^{*} P<0.05\right.$, $\left.{ }^{* *} P<0.01\right)$.

C4H, 4CL, CHS1, CHI, FLS, and ANS genes. Results showed that NtMYB4 significantly reduced the luciferase signal controlled by each promoter, except for that of the ANS gene. In particular, the luciferase signal controlled by the promoter of NtCHS1 were reduced by $88.66 \%$ (Figure $5 \mathrm{~B}$ ). These results indicated that NtMYB4 regulates negatively the transcription of NtCHS1 and thus represses the flavonoid pathway.

\section{NtCHS1 RNAi Silenced Transgenic Plants Showed a Similar Phenotype to NtMYB4 Overexpressing Transgenic Plants Under Salt Stress}

Our previous study revealed that the expression level of NtCHS1 was significantly induced by $200 \mathrm{mM} \mathrm{NaCl}$ treatment (Chen et al., 2017). To validate the function of NtCHS1 in salt stress tolerance, we silenced the gene by RNA interference. The relative expression level of NtCHS1 in the leaves of silenced transgenic tobacco lines was then analyzed using qRT-PCR. Three RNAi silenced lines (CR-1, CR-2, CR-6), out of the five independent NtCHS1-RNAi lines produced, were selected for further analyses, as they showed a significant decrease in the expression level of NtCHS1 compared to WT plants (Figure 6A).

As shown in Supplementary Figure S3B, except for $P A L$ and DFR, the expression of $4 C L, C H I, F 3 H, F L S$, and ANS decreased significantly in NtCHS1 RNAi lines. These lines also displayed a significant decrease in the levels of anthocyanins in the flowers and rutin in the leaves with respect to WT plants (Supplementary Figure S4B and Figure 6B). The results indicated that regulating the expression level of NtCHS1 might alter the expression of major metabolic structural genes and the production of flavonoids in the rutin biosynthesis pathway.

To determine the role of NtCHS1 in salt tolerance, seed germination rate and root length of WT and transgenic tobacco plants cultured on MS medium with or without the addition of $200 \mathrm{mM} \mathrm{NaCl}$ were measured. Severe suppression was observed in RNAi-silenced transgenic seeds, as the germination rate was decreased 95\% under $200 \mathrm{mM} \mathrm{NaCl}$. Moreover, the germination of NtCHS1 RNAi-silenced transgenic seeds was delayed and it was almost abolished in the presence of $200 \mathrm{mM} \mathrm{NaCl}$ (Supplementary Figure S5). For root elongation, there was no obvious difference in seedling growth and root length between WT and transgenic lines under controlled condition (Figure 6D). In the $200 \mathrm{mM} \mathrm{NaCl}$ treatment, significant inhibition of root elongation was observed both in WT and RNAi transgenic lines. In addition, under the above condition RNAi transgenic lines displayed a significant reduction in root length with respect to the WT (Figures 6C,D). Supplementation with $100 \mu$ mol rutin in $200 \mathrm{mM} \mathrm{NaCl} \mathrm{MS}$ medium enhanced root elongation of both WT and NtCHS1 RNAi-silenced transgenic tobacco seedlings significantly (Figures 6C,D). Supplementation with rutin also reduced the ROS levels in the leaves of NtCHS1 RNAi silenced transgenic plants (Figures 6E,F), as in NtMYB4 overexpressing transgenic plants.

\section{NtCHS1 Overexpressing Transgenic Plants Enhanced Salt Tolerance}

To further determine the function of NtCHS1 in tolerance to high salt stress, we overexpressed the NtCHS1 gene in tobacco. Three overexpression lines (CO-2, CO-3, and CO-5) were obtained. The results showed that the expression level of NtCHS1 was significantly increased in the leaves of overexpressing lines compared to WT plants (Figure 7A). We also analyzed the expression levels of the structural genes involved in the flavonoid biosynthetic pathway in NtCHS1 overexpressing lines. As shown in Supplementary Figure S3A, except for $P A L$ with no obvious expression change, the expression of $4 \mathrm{CL}, \mathrm{CHI}$, $F 3 H, D F R, F L S$, and ANS were significantly up-regulated in $N t C H S 1$ overexpressing lines. In addition, rutin content was significantly increased in overexpressing lines (Figure 7B). However, no obvious difference in anthocyanin content was 
A

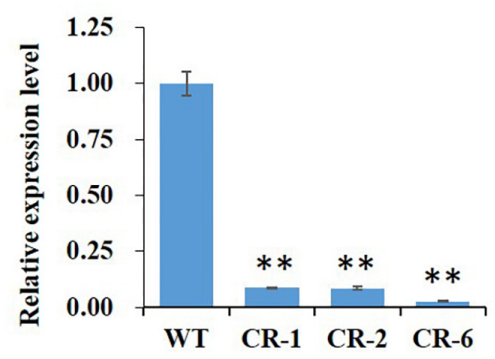

C

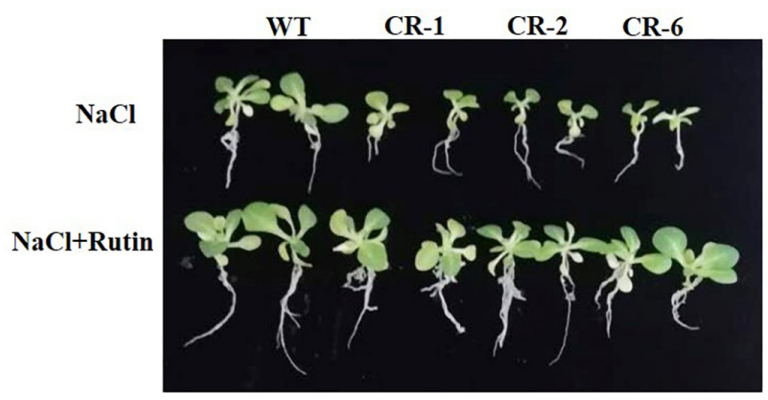

E

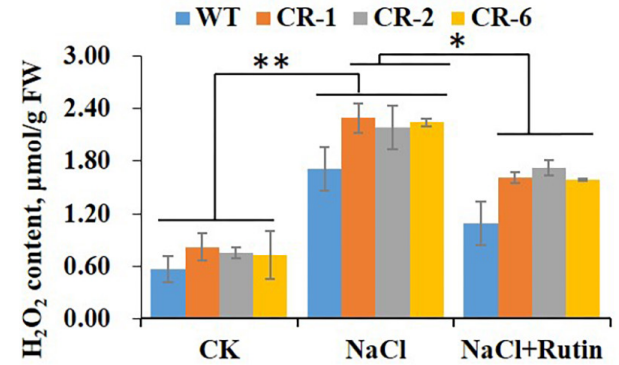

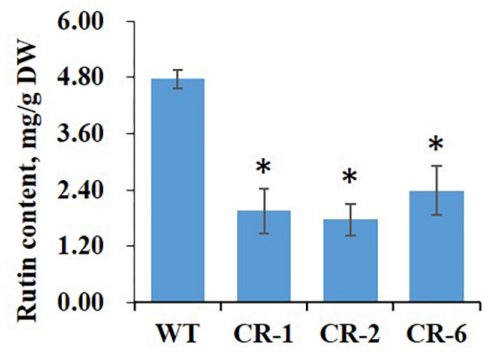

D

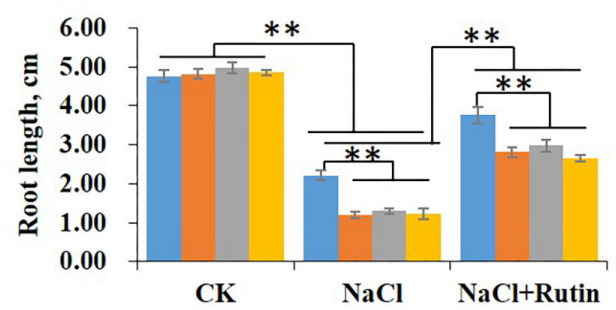

F

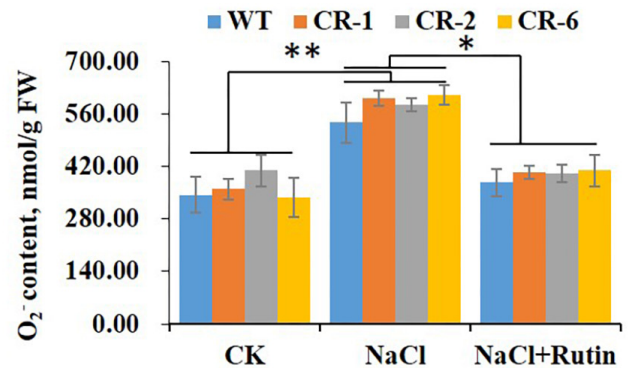

FIGURE 6 | NtCHS1 RNAi silenced transgenic plants show a similar phenotype to NtMYB4 overexpressing transgenic plants in salt stress. (A) The expression level of NtCHS1 decreased in RNAi silenced $\mathrm{T}_{0}$ transgenic tobacco leaves compared to WT. Asterisks in (A) indicate remarkable difference $\left.{ }^{* *} P<0.01\right)$ between NtCHS1 RNAi transgenic lines and WT. (B) Rutin content decreased in NtCHS1 RNAi silenced transgenic tobacco lines compared to WT. DW, dry weight. Asterisks in (B) indicate remarkable difference $\left.{ }^{*} P<0.05\right)$ between NtCHS1 RNAi transgenic lines and WT. (C,D) Root length of WT and NtCHS1 RNAi transgenic plants treated with salt stress with or without exogenous rutin. Values in (D) that are significantly different between treatments and among lines within each treatment are marked with ${ }^{* *} P<0.01$ and ${ }^{*} P<0.05$. (E, $\left.\mathrm{F}\right) \mathrm{H}_{2} \mathrm{O}_{2}$ or $\mathrm{O}_{2}^{-}$content of WT plants and NtCHS1 RNAi silenced transgenic lines under control (CK) and salt treatment (NaCl) with or without rutin supplementation, measured by visible spectrophotometry. FW, fresh weight. Data are expressed as the mean $\pm \mathrm{SD}$ as determined from three independent biological replicates. Values in (E,F) that are significantly different between treatments and among lines within each treatment are marked with ${ }^{* *} P<0.01$ and $* P<0.05$

detected between the three overexpression lines and WT (Supplementary Figure S4A).

The seed germination rate and root length of WT and NtCHS1 overexpressing transgenic tobacco plants were also measured. These parameters did not change between WT and transgenic lines under controlled condition. Under $200 \mathrm{mM} \mathrm{NaCl}$, the germination delayed with respect to the controlled condition in all plants, but the germination rate increased in NtCHS1 overexpressing lines with respect to the control (Supplementary Figure S5). Notably, salt stress induced a significant inhibition of root elongation in both WT (55\%), and, although less markedly $(26 \%)$ in transgenic lines as well (Figures 7C,D). Furthermore, salt stress induced a significant increased in $\mathrm{H}_{2} \mathrm{O}_{2}$ or $\mathrm{O}_{2}^{-}$content in both WT and transgenic tobacco leaves. However, NtCHS1 overexpressing transgenic lines had significant lower $\mathrm{H}_{2} \mathrm{O}_{2}$ or $\mathrm{O}_{2}^{-}$ content than WT plants under salt stress (Figures 7E,F). These results showed that $\mathrm{NtCHS1}$ overexpression caused flavonoid accumulation and affected the rate of ROS scavenging.

\section{DISCUSSION}

In this study, we identified and characterized the NtMYB4 gene from tobacco. Sequence analysis revealed that NtMYB4 contains the R2R3 domain, the zinc-finger domain as well as the EAR and LLsrGIDPxT/SHRxI/L motifs (Supplementary Figure S1), which are typical of sub-group 4 R2R3 MYB repressors (Jin et al., 2000; Zhou et al., 2015). 35S-UAS::GUS leaf transient assay and subcellular analysis showed that NtMYB4 is a nuclear localized transcription repressor in tobacco (Figure 1). Furthermore, dual-luciferase assay verified that NtMYB4 significantly decreased the promoting activity of most 
A
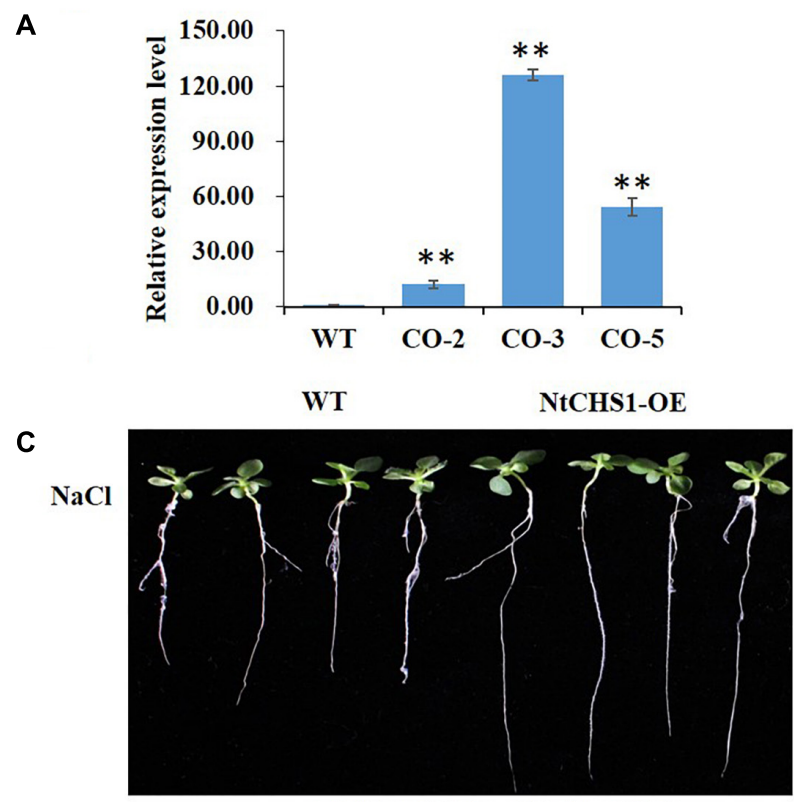

$\backsim \mathrm{WT} \backsim \mathrm{CO}-2 \backsim \mathrm{CO}-3 \backsim \mathrm{CO}-5$

E

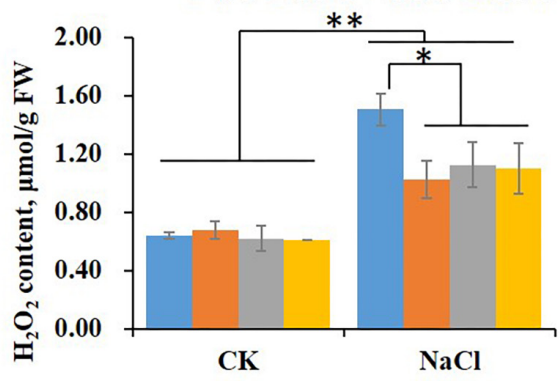

B

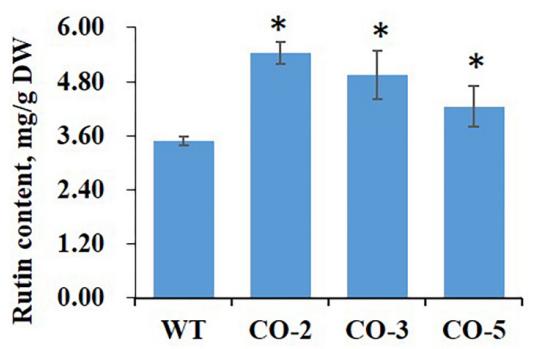

D

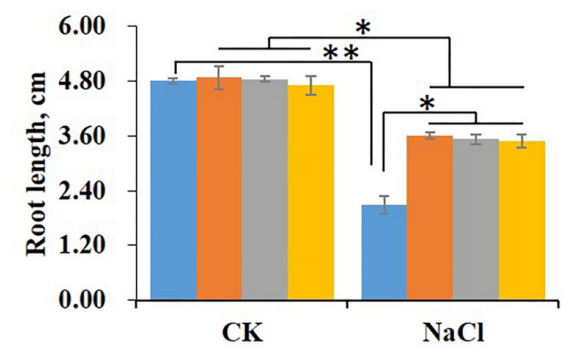

$\backsim \mathrm{WT} \backsim \mathrm{CO}-2 \backsim \mathrm{CO}-3 \backsim \mathrm{CO}-5$

$\mathbf{F}$

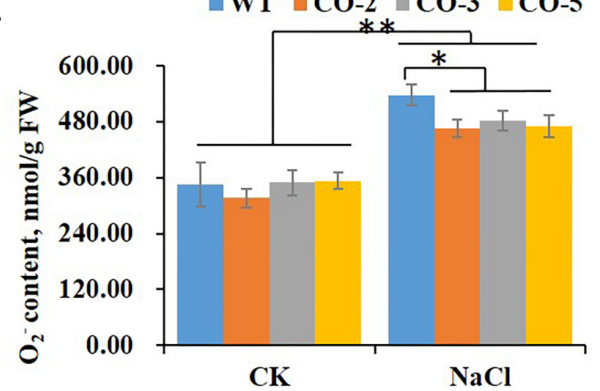

FIGURE 7 | NtCHS1 overexpressing transgenic plants significantly increase salt tolerance. (A) The expression level of NtCHS1 in leaves was significantly increased in overexpressing $T_{0}$ transgenic tobacco leaves compared to WT. Asterisks in (A) indicate remarkable difference (**P $\left.<0.01\right)$ between $N t C H S 1$ overexpressing transgenic lines and WT. (B) Rutin content increased in the leaves of typical NtCHS1 overexpressing transgenic tobacco lines compared to WT. Asterisks in (B) indicate remarkable difference $\left({ }^{*} \mathrm{P}<0.05\right)$ between $\mathrm{NtCHS1}$ overexpressing transgenic lines and WT. (C,D) Root length of WT and NtCHS1 overexpressing transgenic plants (CO-3 line) treated with salt stress. Values in (D) that are significantly different between treatments and among lines within each treatment are marked with ${ }^{* *} P<0.01$ and ${ }^{*} P<0.05$. (E,F) $\mathrm{H}_{2} \mathrm{O}_{2}$ or $\mathrm{O}_{2}^{-}$content of WT plants and NtCHS1 overexpressing transgenic lines under control (CK) and salt treatment $(\mathrm{NaCl})$, measured by visible spectrophotometry. FW, fresh weight. Data are expressed as the mean $\pm \mathrm{SD}$ as determined from three independent biological replicates. Values in (E,F) that are significantly different between treatments and among lines within each treatment are marked with ** $P<0.01$ and $* P<0.05$.

of the key genes in flavonoid biosynthesis, including $P A L, C 4 H$, $4 C L, C H S, C H I$, and FLS (Figure 5B). qRT-PCR results confirmed that NtMYB4 overexpression significantly reduced the expression level of the six key genes (Figure 5A).

The expression of $\mathrm{CHS}$ was negatively regulated by subgroup 4 R2R3 TFs, including AtMYB4, AtMYB7, CsMYB4a, and FtMYB11 (Jin et al., 2000; Fornalé et al., 2014; Li M. et al., 2017; Zhou et al., 2017a). Our results indicated that NtMYB4 directly repressed NtCHS1 gene expression to negatively regulate rutin biosynthesis in tobacco.

Transcription factors are initially vital in sensing salt and their expression levels are changed by salinity, leading to many tolerance responses (Deinlein et al., 2014). Little is known about how subgroup 4 R2R3 MYB TFs function in salt stress. The expression of AtMYB7 was reported to be induced by salinity in A. thaliana, while AtMYB4 expression was not induced (Fornalé et al., 2014; Kim et al., 2015). Here, the expression level of NtMYB4 was found to be highly repressed by salinity (Figure 2A), indicating that NtMYB4 is a salinity-responsive TF. In order to demonstrate whether NtMYB4 functions in salinity tolerance, we overexpressed NtMYB4 (Figure 2B) and investigated plant response to salinity. The results showed that NtMYB4 overexpressing transgenic lines exhibited a much lower seed germination rate and shorter root length than WT plants (Figures $2 \mathbf{C}-\mathbf{F}$ ). In addition, the rutin content was significantly reduced in NtMYB4 overexpressing transgenic lines. Supplementation with exogenous rutin in saline solution improved the salt tolerance of the NtMYB4 overexpressing transgenic lines. These results indicated that inadequate content of rutin might directly result in the salt sensitivity of NtMYB4 overexpressing transgenic lines. 
It is well known that CHS expression is induced by various stimuli, including abiotic and biotic stress (Richard et al., 2000; Zabala et al., 2006; Dao et al., 2011; Chen et al., 2015). In our previous study, we found that NtCHS1 was induced by high salt stress (Chen et al., 2017). Here, we further investigated the functions of NtCHS1 transgenic plants under high salt stress, including overexpression or RNAi-silenced transgenic tobacco lines. Our data showed that NtCHS1 RNAi-silenced transgenic tobacco lines, as NtMYB4 overexpressing transgenic tobacco lines, exhibited reduced rutin contents and salt sensitivity (Figure 6). In addition, NtCHS1 overexpressing transgenic tobacco lines exhibited increased rutin accumulation and higher salt tolerance than WT tobacco (Figure 7). These results further suggest that NtMYB4, by repressing CHS, decreases rutin biosynthesis and, in turn, salt tolerance.

Salinity leads to the overproduction of ROS in plants. ROS are highly reactive and toxic and result in oxidative stress (Gill and Tuteja, 2010). Evidence has accumulated that flavonoids are an important class of antioxidants, and have important roles in plant abiotic stress (Yang et al., 2001; Agati et al., 2012). AtMYB12 has been reported to confer salt and drought tolerance by increasing the levels of flavonoids in transgenic A. thaliana (Wang et al., 2016). Moreover, an extensive integrated analysis of single overexpression of AtMYB75 or AtMYB12, or double overexpression of AtMYB12 and AtMYB75, and tt4 as a flavonoid-deficient mutant, demonstrated that flavonoid overaccumulation was key to enhanced abiotic stress tolerance in Arabidopsis (Nakabayashi et al., 2014). The accumulation of flavonoids is enhanced by salinity (Colla et al., 2013), and the expression of the biosynthetic genes, including $P A L, C H S, C H I$, $D F R, F L S$ and ANS, were shown to be increased in the present study (Supplementary Figure S3A). Similarly moderate salt stress increased rutin accumulation in buckwheat sprout (Lim et al., 2012), which indicated its function in plant salt tolerance. In the present study, higher ROS levels $\left(\mathrm{H}_{2} \mathrm{O}_{2}\right.$ and $\mathrm{O}_{2}^{-}$activity) were detected in NtMYB4 overexpressing (Figures $\mathbf{3 A}, \mathbf{4}$ ) and NtCHS1 RNAi-silenced transgenic tobacco, with reduced rutin content (Figure 6). Supplementation with exogenous

\section{REFERENCES}

Agati, G., Azzarello, E., Pollastri, S., and Tattini, M. (2012). Flavonoids as antioxidants in plants: location and functional significance. Plant Sci. 196, 67-76. doi: 10.1016/j.plantsci.2012.07.014

Ashraf, M., and Harris, P. J. C. (2004). Potential biochemical indicators of salinity tolerance in plants. Plant Sci. 166, 3-16. doi: 10.1016/j.plantsci.2003.10.024

Baâtour, O., Mahmoudi, H., Tarchoun, I., Nasri, N., Trabelsi, N., Kaddour, R., et al. (2013). Salt effect on phenolics and antioxidant activities of Tunisian and Canadian sweet marjoram (Origanum majorana L.) shoots. J. Sci. Food Agric. 93, 134-141. doi: 10.1002/jsfa.5740

Chen, G. H., Sun, J. Y., Liu, M., Liu, J., and Yang, W. C. (2014). SPOROCYTELESS is a novel embryophyte-specific transcription repressor that interacts with TPL and TCP proteins in Arabidopsis. J. Genet. Genomics 41, 617-625. doi: 10.1016/ j.jgg.2014.08.009

Chen, L. J., Guo, H. M., Lin, Y., and Cheng, H. M. (2015). Chalcone synthase EaCHS1 from Eupatorium adenophorum functions in salt stress tolerance in tobacco. Plant Cell Rep. 34, 885-894. doi: 10.1007/s00299-015-1751-7

Chen, S., Pan, X. H., Li, Y. T., Cui, L. J., Zhang, Y. C., Zhang, Z. M., et al. (2017). Identification and characterization of chalcone synthase gene family members rutin reduced $\mathrm{H}_{2} \mathrm{O}_{2}$ and $\mathrm{O}_{2}^{-}$activity in leaves. Moreover, NtCHS1 overexpressing transgenic tobacco increased rutin accumulation and enhanced $\mathrm{O}_{2}^{-}$scavenging ability (Figure 7) under salinity. These results indicated that rutin exhibits strong ROS scavenging ability.

\section{CONCLUSION}

We characterized NtMYB4 functions as a repressor in salt responsiveness. The expression of NtMYB4 was inhibited by salinity, resulting in the activation of NtCHS1 transcription, followed by the accumulation of rutin to scavenge ROS. The results show that rutin is an important antioxidant and is effective in maintaining the balance of ROS in tobacco under salt stress. These results add more light to the roles played by flavonoids in the plant responses to saline stress.

\section{AUTHOR CONTRIBUTIONS}

SC and AY conceived and designed the research. SC, FW, YL, YQ, $\mathrm{XP}, \mathrm{CF}, \mathrm{FL}$, and ZW performed the experiments and analyzed the data. SC and FL wrote the manuscript. YW, AY, and HL support for the research.

\section{FUNDING}

This work was supported by The Agricultural Science and Technology Innovation Program (ASTIP-TRIC01) and China Postdoctoral Science Foundation (2018M630808).

\section{SUPPLEMENTARY MATERIAL}

The Supplementary Material for this article can be found online at: https://www.frontiersin.org/articles/10.3389/fpls.2019.00178/ full\#supplementary-material

in Nicotiana tabacum. J. Plant Growth Regul. 36, 374-384. doi: 10.3389/fpls. 2016.01089

Colla, G., Rouphael, Y., Cardarelli, M., Svecova, E., Rea, E., and Lucini, L. (2013). Effects of saline stress on mineral composition, phenolic acids and flavonoids in leaves of artichoke and cardoon genotypes grown in floating system. J. Sci. Food Agric. 93, 1119-1127. doi: 10.1002/jsfa. 5861

Cui, M. H., Yoo, K. S., Hyoung, S., Nguyen, H. T., Kim, Y. Y., Kim, H. J., et al. (2013). An Arabidopsis R2R3-MYB transcription factor, AtMYB20, negatively regulates type $2 \mathrm{C}$ serine/threonine protein phosphatases to enhance salt tolerance. FEBS Lett. 587, 1773-1778. doi: 10.1016/j.febslet.2013. 04.028

Dao, T. T., Linthorst, H. J., and Verpoorte, R. (2011). Chalcone synthase and its functions in plant resistance. Phytochem. Rev. 10, 397-412. doi: 10.1007/ s11101-011-9211-7

Deinlein, U., Stephan, A. B., Horie, T., Luo, W., Xu, G. H., and Schroeder, J. I. (2014). Plant salt-tolerance mechanisms. Trends Plant Sci. 19, 371-379. doi: 10.1016/j.tplants.2014.02.001

Dixon, R. A., and Paiva, N. L. (1995). Stress-induced phenylpropanoid metabolism. Plant Cell 7, 1085-1097. doi: 10.1105/tpc.7.7.1085 
Dubos, C., Stracke, R., Grotewold, E., Weisshaa, B., Martin, C., and Lepiniec, L. (2010). MYB transcription factors in Arabidopsis. Trends Plant Sci. 15, 573-581. doi: 10.1016/j.tplants.2010.06.005

Fornalé, S., Lopez, E., Salazar-Henao, J. E., Fernández-Nohales, P., Rigau, J., and Caparros-Ruiz, D. (2014). AtMYB7, a new player in the regulation of UVsunscreens in Arabidopsis thaliana. Plant Cell Physiol. 55, 507-516. doi: 10. $1093 / \mathrm{pcp} / \mathrm{pct} 187$

Fornalé, S., Sonbol, F. M., Maes, T., Capellades, M., Puigdomènech, P., Rigau, J., et al. (2006). Down-regulation of the maize and Arabidopsis thaliana caffeic acid O-methyl-transferase genes by two new maize R2R3-MYB transcription factors. Plant Mol. Biol. 62, 809-823. doi: 10.1007/s11103-006-9058-2

Ghorbani, A. (2017). Mechanisms of antidiabetic effects of flavonoid rutin. Biomed. Pharmacother. 96, 305-312. doi: 10.1016/j.biopha.2017.10.001

Gill, S. S., and Tuteja, N. (2010). Reactive oxygen species and antioxidant machinery in abiotic stress tolerance in crop plants. Plant Physiol. Biochem. 48, 909-930. doi: 10.1016/j.plaphy.2010.08.016

He, Y., Li, W., Lv, J., Jia, Y., Wang, M., and Xia, G. (2012). Ectopic expression of a wheat MYB transcription factor gene, TaMYB73, improves salinity stress tolerance in Arabidopsis thaliana. J. Exp. Bot. 63, 1511-1522. doi: 10.1093/jxb/ err389

Horsch, R. F., Fry, J. E., Hoffmann, N. L., Eichholtz, D., Rogers, S. G., and Fraley, R. T. (1985). A simple and general method for transferring genes into plants. Science 227, 1229-1231. doi: 10.1126/science.227.4691.1229

Huang, X., Yao, J., Zhao, Y., Xie, D., Jiang, X., and Xu, Z. (2016). Efficient rutin and quercetin biosynthesis through flavonoids-related gene expression in Fagopyrum tataricum Gaertn. Hairy root cultures with UV-B irradiation. Front. Plant Sci. 7:63. doi: 10.3389/fpls.2016.00063

Jiang, Y., and Deyholos, M. K. (2009). Functional characterization of Arabidopsis $\mathrm{NaCl}$-inducible WRKY25 and WRKY33 transcription factors in abiotic stresses. Plant Mol. Biol. 69, 91-105. doi: 10.1007/s11103-008-9408-3

Jiang, Y., Yang, B., and Deyholos, M. K. (2009). Functional characterization of the Arabidopsis bHLH92 transcription factor in abiotic stress. Mol. Genet. Genomics 282, 503-516. doi: 10.1007/s00438-009-0481-3

Jin, H., Cominelli, E., Bailey, P., Parr, A., Mehrtens, F., Jones, J., et al. (2000). Transcriptional repression by AtMYB4 controls production of UV-protecting sunscreens in Arabidopsis. EMBO J. 19, 6150-6161. doi: 10.1093/emboj/19.22. 6150

Kaur, S., and Muthuraman, A. (2016). Therapeutic evaluation of rutin in twokidney one-clip model of renovascular hypertension in rat. Life Sci. 150, 89-94. doi: 10.1016/j.lfs.2016.02.080

Kerdudo, A., Dingas, A., Fernandez, X., and Faure, C. (2014). Encapsulation of rutin and naringenin in multilamellar vesicles for optimum antioxidant activity. Food Chem. 159, 12-19. doi: 10.1016/j.foodchem.2014.03.005

Kim, J. H., Hyun, W. Y., Nguyen, H. N., Jeong, C. Y., Xiong, L., Hong, S. W., et al. (2015). AtMyb7, a subgroup 4 R2R3 Myb, negatively regulates ABAinduced inhibition of seed germination by blocking the expression of the bZIP transcription factor ABI5. Plant Cell Environ. 38, 559-571. doi: 10.1111/pce. 12415

Ksouri, R., Megdiche, W., Debez, A., Falleh, H., Grignon, C., and Abdelly, C. (2007). Salinity effects on polyphenol content and antioxidant activities in leaves of the halophyte Cakile maritima. Plant Physiol. Biochem. 45, 244-249. doi: 10.1016/j.plaphy.2007.02.001

Kumar, S., Nei, M., Dudley, J., and Tamura, K. (2008). MEGA: a biologistcentric software for evolutionary analysis of DNA and protein sequences. Brief. Bioinform. 9, 299-306. doi: 10.1093/bib/bbn017

Legay, S., Sivadon, P., Blervacq, A. S., Pavy, N., Baghdady, A., Tremblay, L., et al. (2010). EgMYB1, an R2R3 MYB transcription factor from eucalyptus negatively regulates secondary cell wall formation in Arabidopsis and poplar. New Phytol. 188, 774-786. doi: 10.1111/j.1469-8137.2010.03432.x

Li, M., Li, Y., Guo, L., Gong, N., Pang, Y., Jiang, W., et al. (2017). Functional characterization of tea (Camellia sinensis) MYB4a transcription factor using an integrative approach. Front. Plant Sci. 8:943. doi: 10.3389/fpls.2017.00943

Li, P., Li, Y. J., Zhang, F. J., Zhang, G. Z., Jiang, X. Y., Yu, H. M., et al. (2017). The Arabidopsis UDP-glycosyltransferases UGT79B2 and UGT79B3, contribute to cold, salt and drought stress tolerance via modulating anthocyanin accumulation. Plant J. 89, 85-103. doi: 10.1111/tpj.13324

Li, X., Du, Y., Zhang, H., Kuai, Y., Liu, C., and Li, D. (2015). Extracting chlorogenic acid, rutin, nicotine, and solanesol from tobacco. Chin. Tobacco Sci. 36, 1-4.
Li, Y., Pang, T., Shi, J., Lu, X., Deng, J., and Lin, Q. (2014). Liquid chromatography with mass spectrometry method based two-step precursor ion scanning for the structural elucidation of flavonoids. J. Sep. Sci. 37, 3067-3073. doi: 10.1002/jssc. 201400720

Libik, M., Konieczny, R., Pater, B., Slesak, I., and Miszalski, Z. (2005). Differences in the activities of some antioxidant enzymes and in $\mathrm{H} 2 \mathrm{O} 2$ content during rhizogenesis and somatic embryogenesis in callus cultures of the ice plant. Plant Cell Rep. 23, 834-841. doi: 10.1007/s00299-004-0886-8

Lim, J. H., Park, K. J., Kim, B. K., Jeong, J. W., and Kim, H. J. (2012). Effect of salinity stress on phenolic compounds and carotenoids in buckwheat (Fagopyrum esculentum M.) sprout. Food Chem. 135, 1065-1070. doi: 10.1016/ j.foodchem.2012.05.068

Liu, L. J., Zhang, Y. Y., Tang, S. Y., Zhao, Q. Z., Zhang, Z. H., Zhang, H. W., et al. (2010). An efficient system to detect protein ubiquitination by agroinfiltration in Nicotiana benthamiana. Plant J. 61, 893-903. doi: 10.1111/j.1365-313X.2009. 04109.x

Liu, S., Ju, J., and Xia, G. (2014). Identification of the flavonoid $3^{\prime}$-hydroxylase and flavonoid $3^{\prime}, 5^{\prime}$-hydroxylase genes from Antarctic moss and their regulation during abiotic stress. Gene 543, 145-152. doi: 10.1016/j.gene.2014. 03.026

Maloney, G. S., DiNapoli, K. T., and Muday, G. K. (2014). The anthocyanin reduced tomato mutant demonstrates the role of flavonols in tomato lateral root and root hair development. Plant Physiol. 166, 614-631. doi: 10.1104/pp.114.240507

Moore, J. P., Westall, K. L., Ravenscroft, N., Farrant, J. M., Lindsey, G. G., and Brandt, W. F. (2005). The predominant polyphenol in the leaves of the resurrection plant Myrothamnus flabellifolius, 3,4,5 tri-O-galloylquinic acid, protects membranes against desiccation and free radical-induced oxidation. Biochem. J. 385, 301-308. doi: 10.1042/BJ20040499

Nakabayashi, R., Yonekura-Sakakibara, K., Urano, K., Suzuki, M., Yamada, Y., Nishizawa, T., et al. (2014). Enhancement of oxidative and drought tolerance in Arabidopsis by overaccumulation of antioxidant flavonoids. Plant J. 77, 367-379. doi: 10.1111/tpj.12388

Pandey, A., Misra, P., Choudhary, D., Yadav, R., Goel, R., Bhambhani, S., et al. (2015). AtMYB12 expression in tomato leads to large scale differential modulation in transcriptome and flavonoid content in leaf and fruit tissues. Sci. Rep. 5:12412. doi: 10.1038/srep 12412

Pattanaik, S., Kong, Q., Zaitlin, D., Werkman, J. R., Xie, C. H., Patra, B., et al. (2010). Isolation and functional characterization of a floral tissue-specific R2R3 MYB regulator from tobacco. Planta 231, 1061-1076. doi: 10.1007/s00425-010-1108y

Richard, S., Lapointe, G., Rutledge, R. G., and Séguin, A. (2000). Induction of chalcone synthase expression in white spruce by wounding and jasmonate. Plant Cell Physiol. 41, 982-987. doi: 10.1093/pcp/pcd017

Torun, H. (2018). Time-course analysis of salicylic acid effects on ROS regulation and antioxidant defense in roots of hulled and hulless barley under combined stress of drought, heat and salinity. Physiol. Plant. doi: 10.1111/ppl.12798 [Epub ahead of print].

Tuteja, N. (2007). Chapter twenty-four-mechanisms of high salinity tolerance in plants. Methods Enzymol. 428, 419-438. doi: 10.1016/S0076-6879 (07)28024-3

Wang, F., Kong, W., Wong, G., Fu, L., Peng, R., Li, Z., et al. (2016). AtMYB12 regulates flavonoids accumulation and abiotic stress tolerance in transgenic Arabidopsis thaliana. Mol. Genet. Genomics 291, 1545-1559. doi: 10.1007/ s00438-016-1203-2

Watkins, J. M., Chapman, J. M., and Muday, G. K. (2017). Abscisic acid-induced reactive oxygen species are modulated by flavonols to control stomata aperture. Plant Physiol. 175, 1807-1825. doi: 10.1104/pp.17.01010

Winkel-Shirley, B. (2001). Flavonoid biosynthesis. A colorful model for genetics, biochemistry, cell biology, and biotechnology. Plant Physiol. 126, 485-493. doi: 10.1104/pp.126.2.485

Xu, P. S., Bai, J. F., Liu, J. W., and Li, H. G. (2011). Construction of LSV and LMoV binary virus resistant RNAi vector using gateway technology. Chin. Agric. Sci. Bull. 27, 144-147.

Yang, B., Kotani, A., Arai, K., and Kusu, F. (2001). Estimation of the antioxidant activities of flavonoids from their oxidation potentials. Anal. Sci. 17, 599-604. doi: 10.2116/analsci.17.599

Yang, O., Popova, O. V., Süthoff, U., Lüking, I., Dietz, K. J., and Golldack, D. (2009). The Arabidopsis basic leucine zipper transcription factor AtbZIP24 regulates 
complex transcriptional networks involved in abiotic stress resistance. Gene 436, 45-55. doi: 10.1016/j.gene.2009.02.010

Yoo, H., Ku, S. K., Baek, Y. D., and Bae, J. S. (2014). Anti-inflammatory effects of rutin on HMGB1-induced inflammatory responses in vitro and in vivo. Inflamm. Res. 63, 197-206. doi: 10.1007/s00011-0130689-x

Yu, O., and Jez, J. M. (2008). Nature's assembly line: biosynthesis of simple phenylpropanoids and polyketides. Plant J. 54, 750-762. doi: 10.1111/j.1365313X.2008.03436.x

Zabala, G., Zou, J., Tuteja, J., Gonzalez, D. O., Clough, S. J., and Vodkin, L. O. (2006). Transcriptome changes in the phenylpropanoid pathway of Glycine max in response to Pseudomonas syringae infection. BMC Plant Biol. 6:26. doi: 10.1186/1471-2229-6-26

Zhang, H., Han, B., Wang, T., Chen, S., Li, H., Zhang, Y., et al. (2012). Mechanisms of plant salt response: insights from proteomics. J. Proteome Res. 11, 49-67. doi: $10.1021 / \mathrm{pr} 200861 \mathrm{w}$

Zhang, L., Wang, Y., Sun, M., Wang, J., Kawabata, S., and Li, Y. (2014). BrMYB4, a suppressor of genes for phenylpropanoid and anthocyanin biosynthesis, is down-regulated by UV-B but not by pigment-inducing sunlight in turnip cv. Tsuda. Plant Cell Physiol. 55, 2092-2101. doi: 10.1093/pcp/ pcu 137

Zhang, W., Yang, G., Mu, D., Li, H., Zang, D., Xu, H., et al. (2016). An ethylene-responsive factor BpERF11 negatively modulates salt and osmotic tolerance in Betula platyphylla. Sci. Rep. 6:23085. doi: 10.1038/ srep23085

Zhao, Q., Ren, Y. R., Wang, Q. J., Wang, X. F., You, C. X., and Hao, Y. J. (2016). Ubiquitination-related MdBT scaffold proteins target a bHLH transcription factor for iron homeostasis. Plant Physiol. 172, 1973-1988. doi: 10.1104/pp.16. 01323

Zhou, M., Sun, Z., Ding, M., Logacheva, M. D., Kreft, I., Wang, D., et al. (2017a). FtSAD2 and FtJAZ1 regulate activity of the FtMYB11 transcription repressor of the phenylpropanoid pathway in Fagopyrum tataricum. New Phytol. 216, 814-828. doi: 10.1111/nph.14692

Zhou, M., Zhang, K., Sun, Z., Yan, M., Chen, C., Zhang, X., et al. (2017b). LNK1 and LNK2 corepressors interact with the MYB3 transcription factor in phenylpropanoid biosynthesis. Plant Physiol. 174, 1348-1358. doi: 10.1104/pp. 17.00160

Zhou, M., Sun, Z., Wang, C., Zhang, X., Tang, Y., Zhu, X., et al. (2015). Changing a conserved amino acid in R2R3-MYB transcription repressors results in their cytoplasmic accumulation and abolishes their repressive activity in Arabidopsis. Plant J. 84, 395-403. doi: 10.1111/tpj. 13008

Conflict of Interest Statement: The authors declare that the research was conducted in the absence of any commercial or financial relationships that could be construed as a potential conflict of interest.

Copyright (c) 2019 Chen, Wu, Li, Qian, Pan, Li, Wang, Wu, Fu, Lin and Yang. This is an open-access article distributed under the terms of the Creative Commons Attribution License (CC BY). The use, distribution or reproduction in other forums is permitted, provided the original author(s) and the copyright owner(s) are credited and that the original publication in this journal is cited, in accordance with accepted academic practice. No use, distribution or reproduction is permitted which does not comply with these terms. 